\title{
Reply to discussions on "Applying fuzzy theory and genetic algorithm to interpolate precipitation" by Zekai Sen
}

\author{
C.L. Chang ${ }^{a, *}$, S.L. Lo ${ }^{b}$, S.L. Yu ${ }^{c}$
}

a Department of Environmental Resources Management, Chia Nan University of Pharmacy \& Science, Tainan, Taiwan, ROC

b Graduate Institute of Environmental Engineering, National Taiwan University, Taipei 106, Taiwan, ROC

c Department of Civil Engineering, University of Virginia, Charlottesville, VA, USA

Received 21 February 2006; received in revised form 6 May 2006; accepted 16 June 2006

\section{KEYWORDS \\ Discussion; \\ Fuzzy theory; \\ Genetic algorithm; \\ Precipitation interpolation}

\begin{abstract}
Summary Zekai Sen provides several comments on our paper (Chang, C.L., Lo, S.L., Yu, S.L., 2005. Applying fuzzy theory and genetic algorithm to interpolate precipitation. J. Hydrol. 314, 92-104). More discussions could create different opinions and ideas, and they are all very significant for the development of methodology or study application. However, Sen has some misunderstanding on our paper. The following discussions follow the order of Sen's comments. (c) 2006 Elsevier B.V. All rights reserved.
\end{abstract}

\section{The inverse distance method}

Sen considers that the weighting factors presented in our paper are only based on geometrical concept of inverse distance method, whereas flexibility in the weighting factors should include the effects of precipitation amounts recorded in each gauge. Sen refers to some drawbacks of the weighting functions, which do not take into consideration the natural variability of meteorological phenomenon (Cressman, 1959; Sasaki, 1960; Barnes, 1964; Thiebaux and Pedder, 1987). Sen also states "The weighing functions

\footnotetext{
* Corresponding author. Tel.: +886 2 23625373; fax: +886 2 23628764.

E-mail address: f89541201@ntu.edu.tw (C.L. Chang).
}

must reflect the behavior of the meteorological phenomena in addition to the station configuration geometry."' Certainly, the meteorological phenomenon would also have some influence on weighting functions. However, no literature could accurately predict or control meteorological phenomenon. Therefore, it is valuable to quantify the weighting factors of each rainfall gauge on the basis by taking into consideration the station configuration geometry. Our paper introduces a new methodology for adjusting the weighting factors of each rainfall gauge to represent the relative influence in precipitation estimation. Even though it is not the optimal methodology for estimating precipitation, the estimation error of precipitation is definitely lowered by this method developed in our study, compared with traditional methods. 


\section{Fuzzy theory and GA}

Sen deems that it is not valid to express the weighting factors in the IDW interpolation method and the membership function in the fuzzy theory with the same idea in different words. However, the relative influence of each surrounding rainfall gauge is an abstract factor, so it is not easy to use absolutely binary classification, i.e. completely related and completely unrelated classes. Thus, it is valuable to apply fuzzy logic for addressing these situations by allowing variables to be "partially true" and/or "partially false" (Abdel-Kader et al., 1998). IDW method does the same way to describe the relative influence of each rainfall gauge. The effect of site configuration and elevation-difference variability of rainfall stations is considered in our paper to decide the weighting factors, and the fuzzy membership functions is clearly defined for representing the weighting factors.

Sen has some misunderstanding on the definition of the methodology developed in our paper. Our paper explains that "Although the tendencies of these two membership functions are resemble, the scales of horizontal distances and elevation differences are different. In this study, normalization technology (Craig and Karen, 1995) was applied for avoiding the misleading analysis due to various scales of horizontal distances and elevation differences. The elements in set $U$, and $V$ are redefined as normalized horizontal distances and elevation differences" (Chang et al., 2005). The variable " $d$ ", horizontal distances, and the variable " $h$ ", elevation differences, are transformed to the same scale without any unit by normalization procedure. The values are fixed between 1 and 10. The calculation of transforming a parameter with unit to a normalized dimensionless value can be shown as following equations:

$d^{\prime}=1+9\left(\frac{d-d_{\min }}{d_{\max }-d_{\min }}\right)$

$h^{\prime}=1+9\left(\frac{h-h_{\min }}{h_{\max }-h_{\min }}\right)$

where $d^{\prime}$ and $h^{\prime}$ are normalized $d$ and $h$ separately. When $d \rightarrow 0$ (or $h \rightarrow 0$ ), $d^{\prime} \rightarrow 1$ (or $h^{\prime} \rightarrow 1$ ) and the membership functions, $\mu_{\mathrm{pd}}(d)$ and $\mu_{\mathrm{ph}}(h)$, would close to 1 as well. Since $d^{\prime}$ and $h^{\prime}$ are always bigger than (or equal to) 1 and $m$ and $n$ are bigger than 0 , the membership functions are always less than (or equal to) 1. The definition of the methodology is reasonable and logical.

Moreover, Sen states "It is not clear why minimum and multiplication operations are adopted for better solution". The results are searched by GA procedure, and Figs. 6 and 7 in our paper can obviously demonstrate the statement. If Sen could carefully ruminate our paper, he would have more clear understanding.

\section{Applying fuzzy sets to interpolate precipitation}

Although our paper does not consider the meteorological phenomena in the allotment of weighting factors for each rainfall gauge, it combines the effect of horizontal and elevation differences between each rainfall station. The results verify that the consideration of the influencing factor of elevation difference could lower the estimation error.
For instance, the differences between the elevations of the Feitsui rainfall station and its nearby gauges are large and various, and the results show that the estimation error is much more reduced by applying the method developed in this work based on the fuzzy theory than other methods, which do not take into consideration of elevation-difference variability (Chang et al., 2005).

Again, Sen states "All the discussions concerning the estimation errors are related to horizontal distances, which are static and do not change with time, but there is no mention about the rainfall amount variations that are significant from hydrology point of view' '. Rainfall characteristic might be an effect for estimating precipitation. The methods, which only consider station configuration geometry, always assume that the difference of rainfall amount is influenced by the site configuration or elevation differences between each rainfall gauge. This assumption is reasonable, even though sometimes it is not absolutely true. Therefore, it is still valuable to take into consideration the station configuration geometry only, because the meteorological phenomenon cannot be easily controlled. The results in our work show that the estimated error of precipitation at Taiping rainfall station cannot be effectively reduced by any method, because the average horizontal distance between the Taiping rainfall gauge and its nearby rainfall stations is so large (Chang et al., 2005). It shows that the methodology, which only considers the site configuration, could result in some errors, when the assumption mentioned above is not valid. The methodology developed in our study is, of course, not perfect or the best one. However, it certainly improves the traditional methods in the consideration of the effect of site configuration and elevation-difference variability.

Moreover, Sen has a query about the word "peak" used in our paper. It is a tiny mistake on the statement. The word "valley" is definitely much better for presenting the minimum error point than the word "peak". Sen's suggestion is highly appreciated.

\section{Conclusions}

Some unclear statements have been explained herein. Sen emphasizes the importance of meteorological phenomenon in precipitation estimation. However, our study focuses on the improvement of the consideration of the effects of station configuration geometry, and combines the effects of horizontal and elevation differences by the concept of fuzzy membership functions. Although Sen does not consider that the weighting factors in the IDW method and fuzzy membership functions are in the same idea, we still insist the important opinion and application in our work. The achievements and innovation of the methodology development is significant, even though a new method could not be a perfect one.

\section{References}

Abdel-Kader, M.G., Dugdale, D., Taylor, P., 1998. Investment Decisions in Advanced Manufacturing Technology - A Fuzzy Set Theory Approach. Ashgate Publishing Company, Burlington, VT.

Barnes, S.L., 1964. A technique for maximizing details in numerical weather map analysis. J. Appl. Meteorol. 3, 396-409.

Chang, C.L., Lo, S.L., Yu, S.L., 2005. Applying fuzzy theory and genetic algorithm to interpolate precipitation. J. Hydrol. 314, 92-104. 
Craig, E.H., Karen, A.K., 1995. To normalize or not to normalize? Fat is the question. Environ. Toxicol. Chem. 14 (5), 801-807.

Cressman, G.P., 1959. An operational objective analysis system. Mon. Weather Rev. 87 (10), 367-374.
Sasaki, Y., 1960. An objective analysis for determining initial conditions for the primitive equations. Tech. Rep. (Ref.60-16T), Texas A/M University, College Station.

Thiebaux, H.J., Pedder, M.A., 1987. Spatial Objective Analysis. Academic Press, New York, p. 299. 